Supporting Information on

\title{
Self-assembled templates for polypeptide synthesis
}

Maxim G. Ryadnov ${ }^{1}$ and Derek N. Woolfson ${ }^{1,2}$

${ }^{1}$ School of Chemistry, University of Bristol, Cantock's Close, Bristol BS8 1TS, UK

${ }^{2}$ Department of Biochemistry, School of Medical Sciences, University Walk, Bristol, BS8 1TD, UK

\section{Abbreviations}

Boc - tert-butoxycarbonyl; DIPEA - diisopropylethylamine; EDC - ethyl-3-[3dimethylaminopropyl]carbodiimide hydrochloride; ESI - ElectroSpray ionization; Fmoc - 9Fluorenylmethoxycarbonyl; HBTU - $O$-Benzotriazole- $N, N, N^{\prime}, N^{\prime}$-tetramethyl-uronium-hexafluorophosphate; HEPES - N-2-hydroxyethylpiperazine- $N$ '-2-ethane sulfonic acid; RP-HPLC - reversed phase high pressure liquid chromatography; MALDI - Matrix-assisted laser desorption/ionization; MOPS - (3(N-morpholino)propanesulfonic acid; PEO - poly(ethyleneoxide); TIS - triisopropyl silane; TFA trifluoroacetic acid

\section{Additional figures:}


Spectrum Report

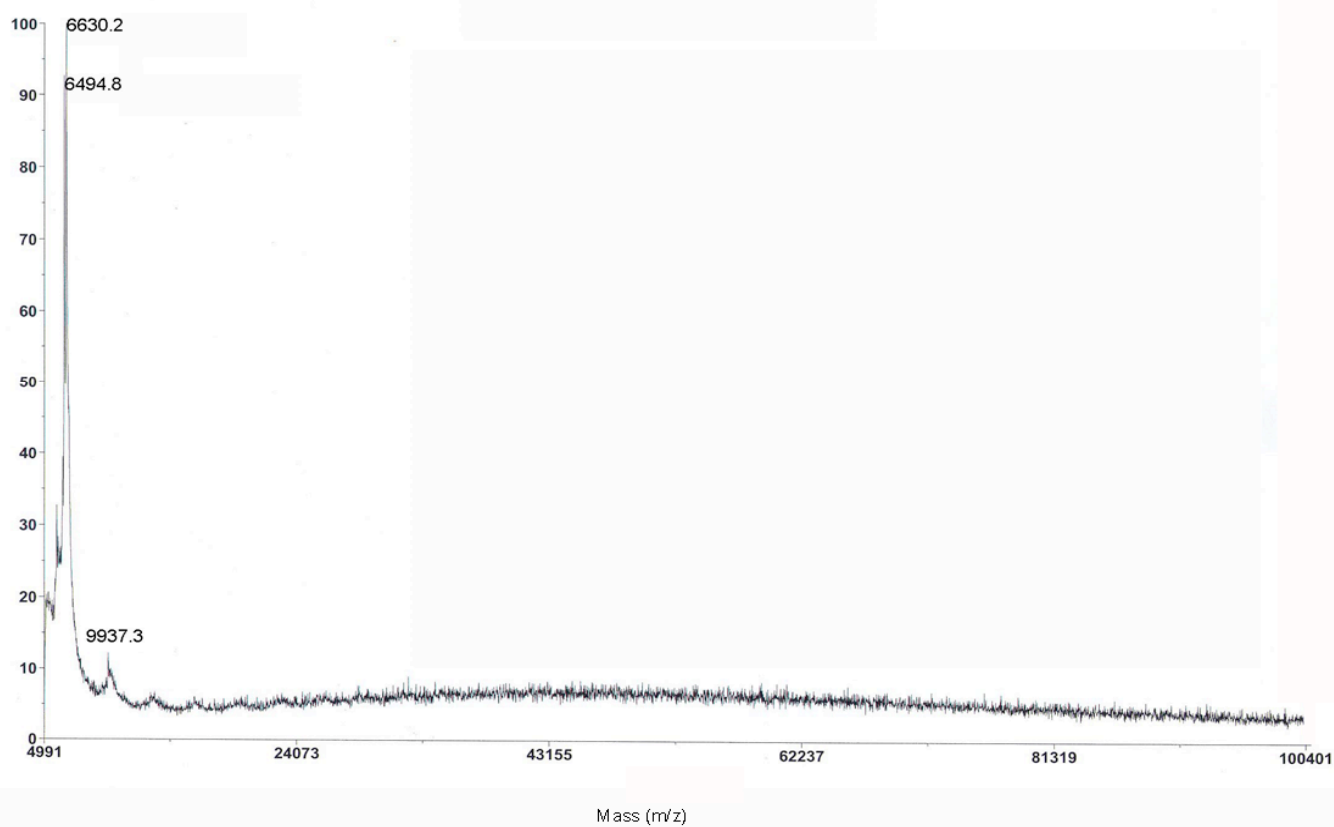

Spectrum Report

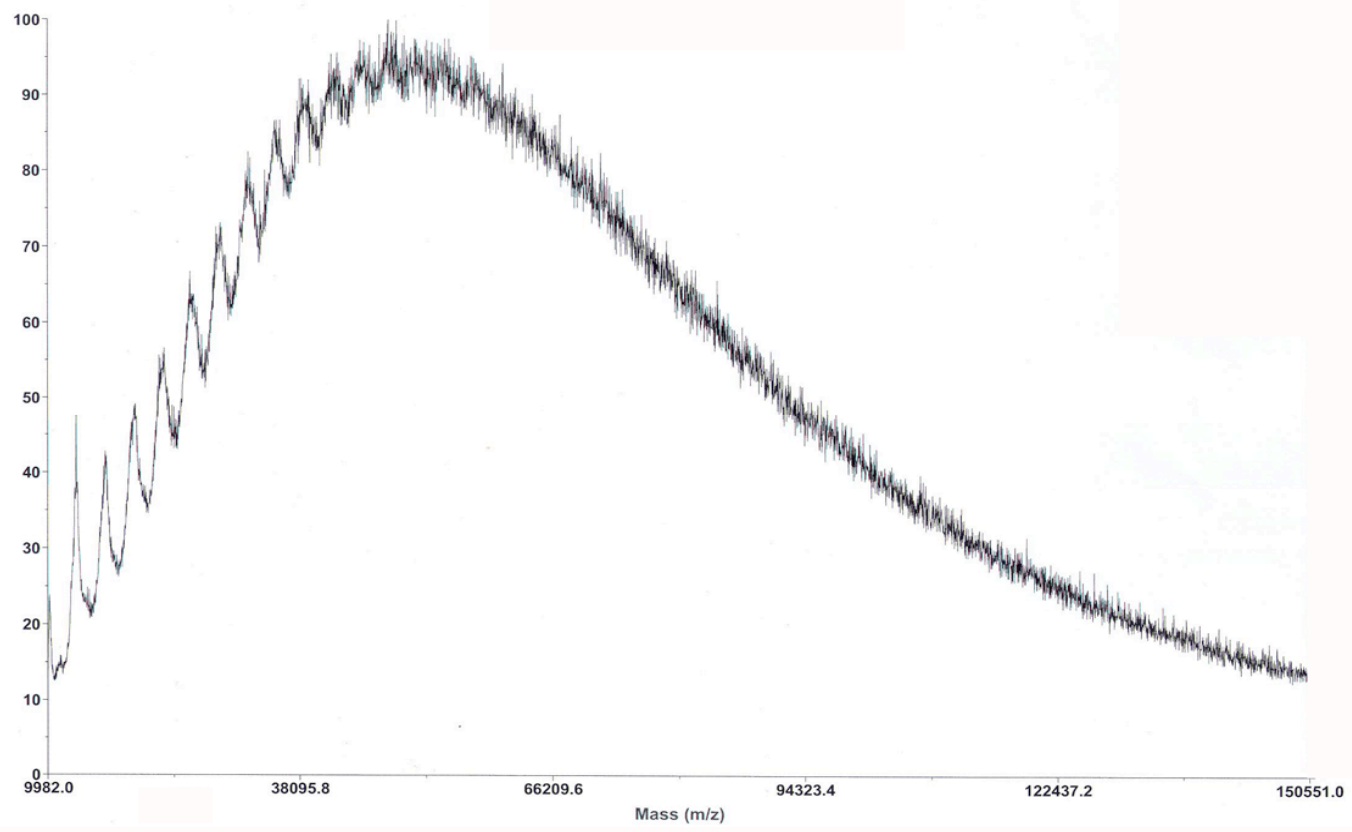




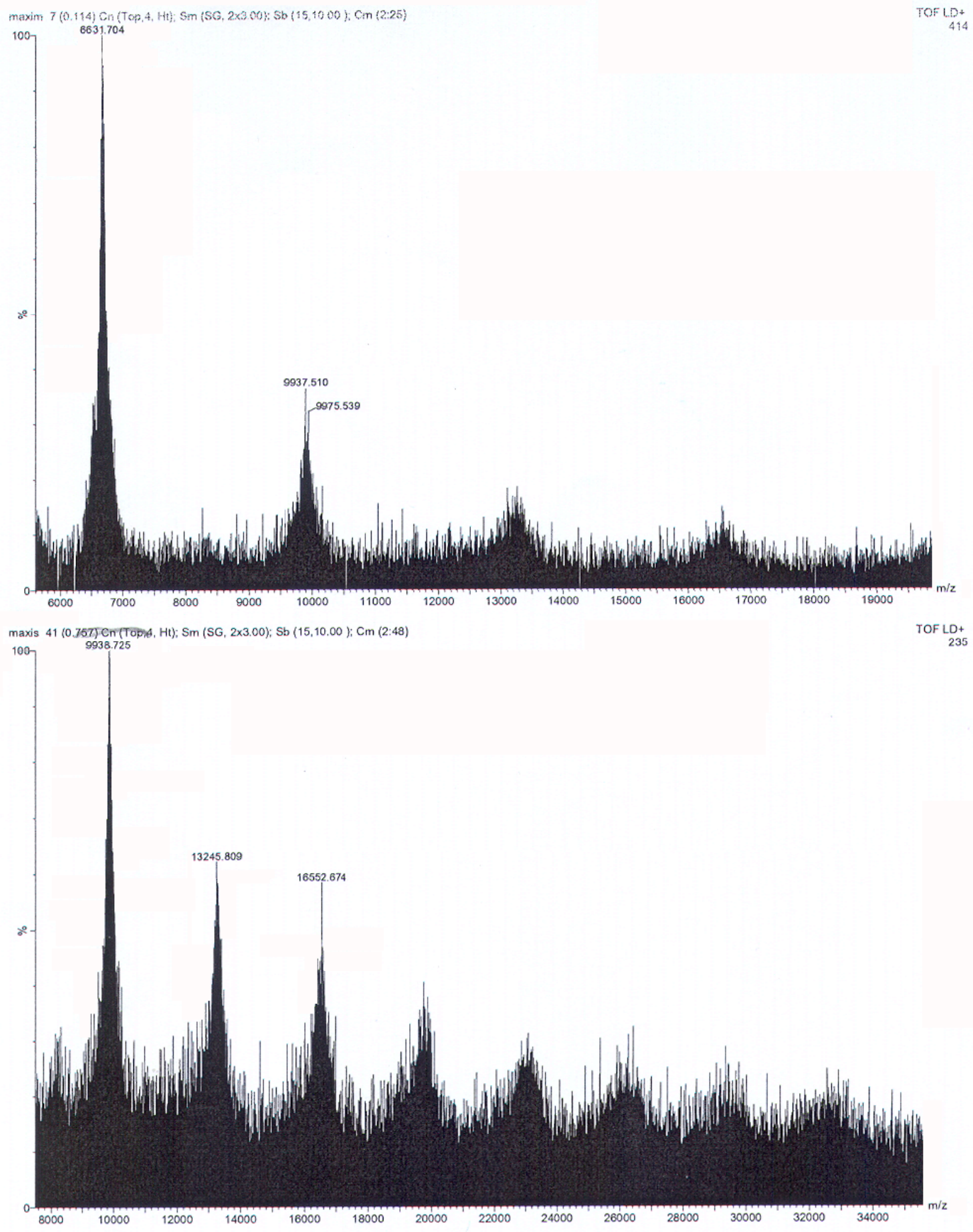

Figure S1. Two sets of raw mass-spectra for SAFE assembled from SAF-p2E - SAFs in 1:100 ratios. Spectra two and four (from top) are high $\mathrm{m} / \mathrm{z}$ cuts of the whole range spectra with dominating low molecular weight peaks (first and third from top). 
Spectrum Report

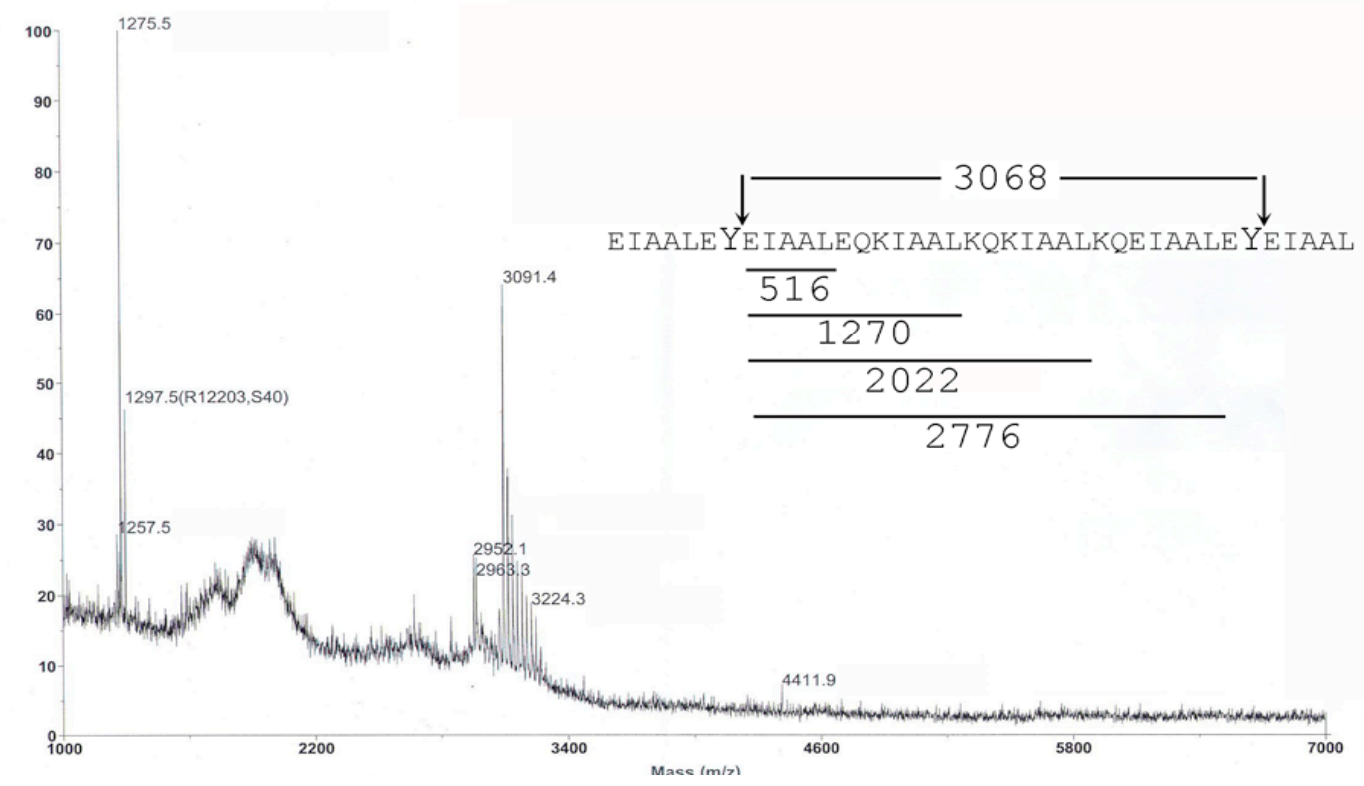

Figure S2. Raw mass-spectrum for chymotrypsin-digested STePE. 

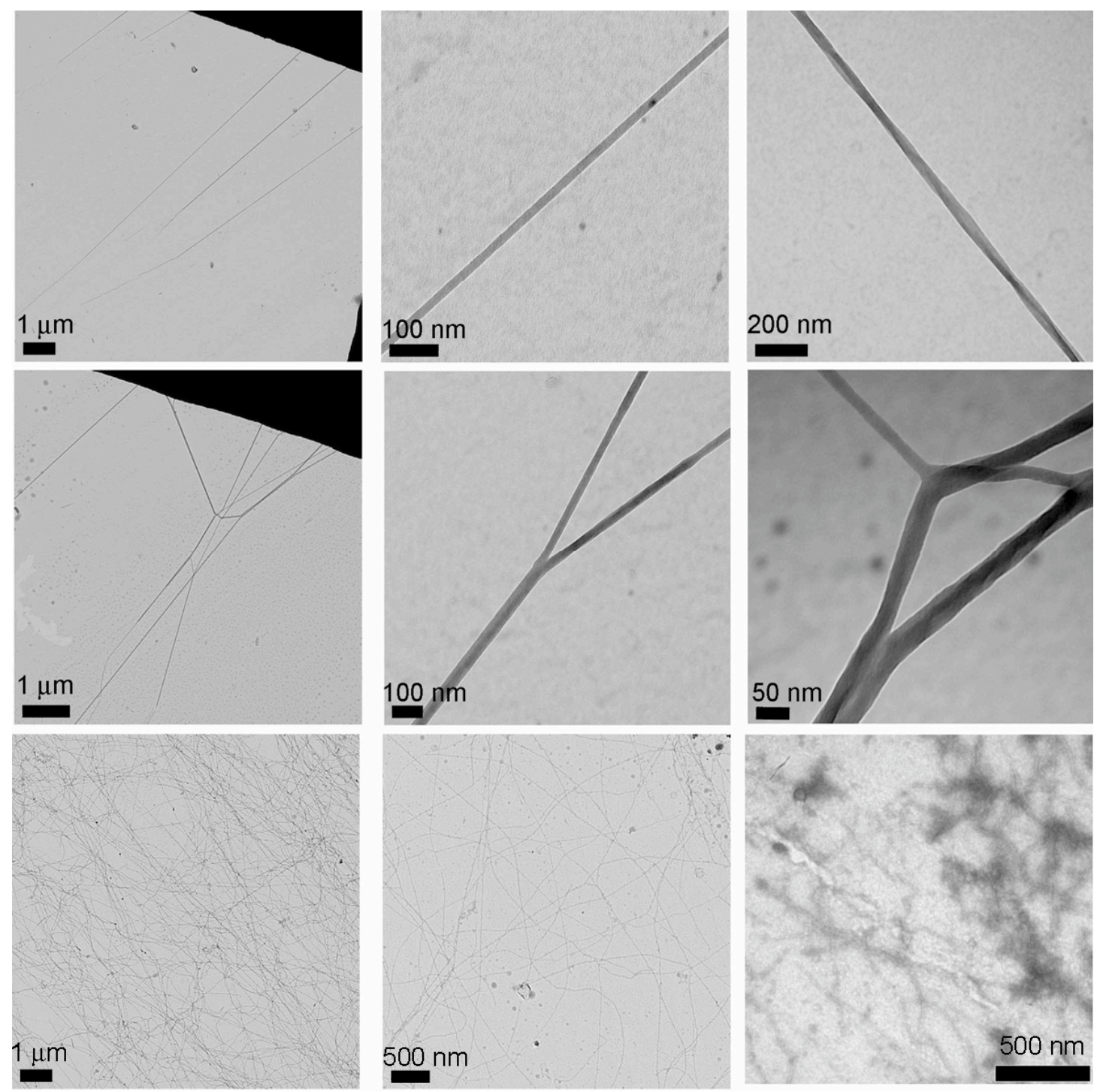

Figure S3. Electron micrographs of different morphologies for SAFE. Straight (top panel) and networked (middle panel) fibers at neutral $\mathrm{pH}$ and ligated peptides bundled into entangled fibrils at $\mathrm{pH}$ 8-9 (bottom panel). Ligated peptides assembled from SAF-p2E -SAFs in 1:100 ratios (bottom right). 

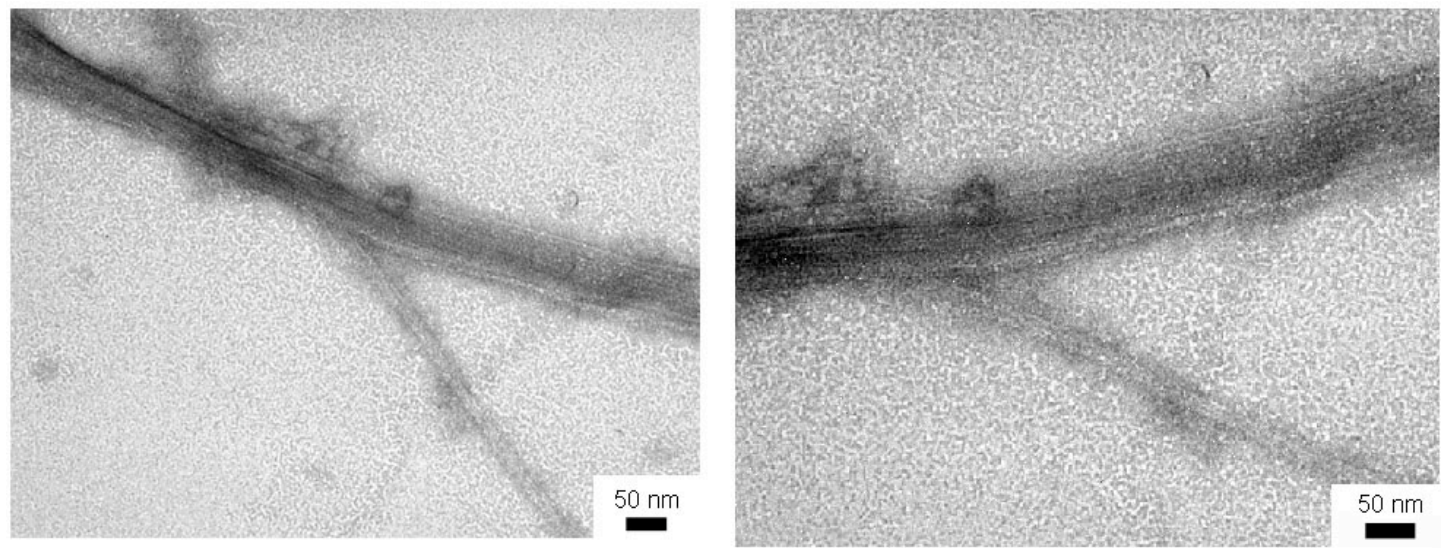

Figure S4. Electron micrographs of STeP with distinct thinner bundles of individual fibrils.
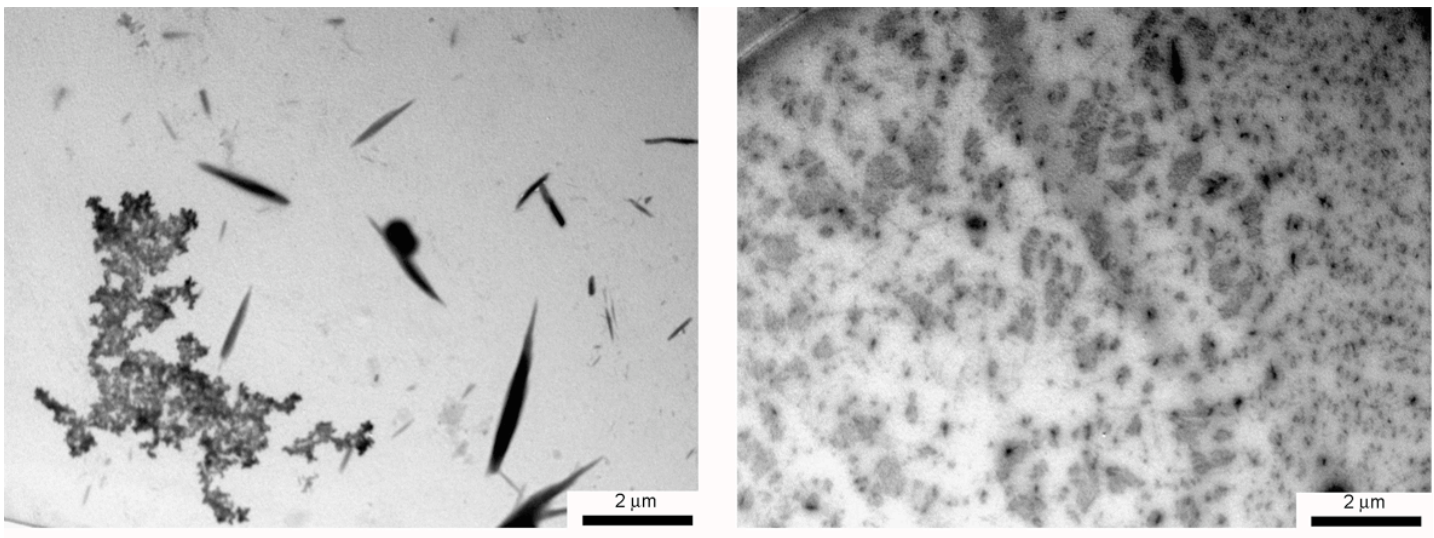

Figure S5. Electron micrographs of SAFE assembled from acSAF-p1E:SAF-p1:SAF-p2 in 0.01:1:1 ratio at $\mathrm{pH} 7$ (left) and $\mathrm{pH} 8$ (right). 